\title{
The effects of deep emotions on chronic fatigue in young adults
}

Seishu Nakagawa ${ }^{1,2^{*}}$, Hikaru Takeuchi ${ }^{2}$, Yasuyuki Taki ${ }^{2}$, Rui Nouchi ${ }^{2}$, Yuka Kotozaki ${ }^{2}$ Takamitsu Shinada ${ }^{2}$, Tsukasa Maruyama ${ }^{2}$, Atsushi Sekiguchi², 3, , Kunio Iizuka²

Ryoichi Yokoyama ${ }^{4}$, Yuki Yamamoto ${ }^{2}$, Sugiko Hanawa ${ }^{2}$, Tsuyoshi Araki ${ }^{5}$ Carlos Makoto Miyauchi ${ }^{2}$, Daniele Magistro ${ }^{6}$, Kohei Sakaki ${ }^{2}$, Hyeonjeong Jeong ${ }^{2}$ Yukako Sasaki $^{2}$, Ryuta Kawashima ${ }^{2}$

\section{Tohoku Medical and Pharmaceutical University, Sendai, Japan \\ 2 Tohoku University, Sendai, Japan 3 National Center of Neurology and Psychiatry, Japan 4 Kobe University, Japan 5 ADVANTAGE Risk Management Co., Ltd, Tokyo, Japan 6 Nottingham Trent University, $U K \quad *$ Email: seishu@tohoku-mpu.ac.jp}

\section{Introduction}

Chronic fatigue (not relieved by rest) is a complex conscious sensation and emotion affected by deep emotions. Perfectionism $\Rightarrow$ Enormous energy $\Rightarrow$ Depression, social phobia, eating disorders, and obsessive-compulsive Perfectionism (Shafran et al, 2001) $\Rightarrow$ Chronic fatigue?

Loneliness and hostility $\Rightarrow$ Stress $\Rightarrow$ Cumulated stress $\Rightarrow$ Chronic fatigue (Jaremka et al, 2014) .

$>$ General self-efficacy was negatively associated with chronic fatigue (Wang et al, 2017).

$\Rightarrow$ Hypothesis: Chronic fatigue regression model using deep emotions

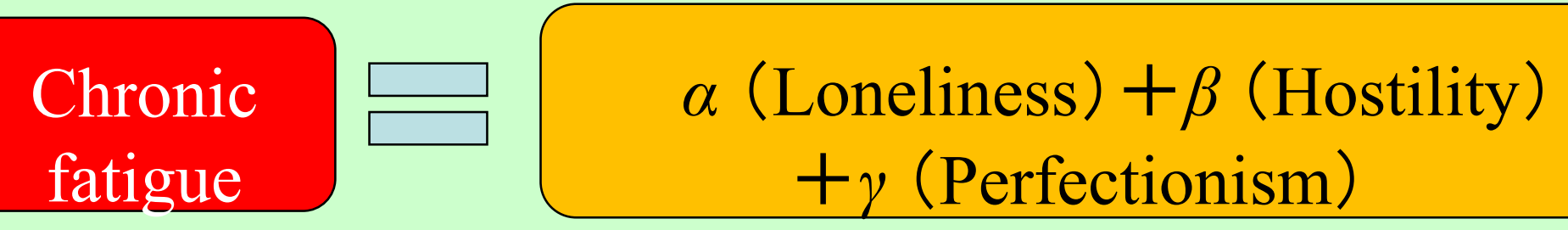

Purpose: To test the hypothesis and elucidate the effects of deep emotions on chronic fatigue.

\section{Methods}

$>$ Subjects: Healthy young volunteers in University student $(N=767)$

$>$ Assessment: Increasing scores on each scale mean more tendencies to the deep emotions.

Chronic fatigue: Checklist individual strength (CIS) ( $76>$ : Chronic fatigue)

- General self efficacy: General self-efficacy scale (GSES)

- Loneliness: UCLA loneliness scale

- Hostility and Perfectionism: the Coronaryprone Type Scale for Japanese.

$>$ Analyses:

ANOVA for gender differences.

- Pearson correlations among the psychological scores.

Multiple stepwise regression analysis with CIS scores as dependent variables and other scores as independent variables.

$>$ No conflict of interest. The procedures were approved by the Ethics Committee of Tohoku University. Written informed consent was obtained from each subject in accordance with the Declaration of Helsinki.

Results

\begin{tabular}{|l|l|l|l|l|l|}
\hline Measure & Total (SD) & Males (423) & Females (344) & $\boldsymbol{P}$ & $\boldsymbol{F}$ \\
\hline CIS & $69.1(17.8)$ & $70.0(17.6)$ & $67.9(17.9)$ & 0.096 & 2.78 \\
\hline GSES & $69.7(12.4)$ & $69.3(13.0)$ & $70.2(11.7)$ & 0.295 & 1.10 \\
\hline Loneliness & $35.3(8.6)$ & $36.4(9.0)$ & $33.8(7.8)$ & $<0.001^{* *}$ & 18.9 \\
\hline Hostility & $22.2(8.3)$ & $22.9(8.3)$ & $21.3(8.1)$ & $0.005^{*}$ & 7.8 \\
\hline Perfectionism & $35.7(7.2)$ & $36.5(7.2)$ & $34.7(7.2)$ & $<0.001^{* *}$ & 11.4 \\
\hline
\end{tabular}

Correlations of chronic fatigue with deep emotions

\begin{tabular}{|c|c|c|c|c|}
\hline & CIS & GSES & Loneliness & Hostility \\
\hline GSES & $-0.595^{* *}$ & & \multicolumn{2}{|c|}{${ }^{*} P<0.01$} \\
\hline Loneliness & $0.507^{* *}$ & $-0.543^{* *}$ & \multicolumn{2}{|c|}{${ }^{* *} P<0.001$} \\
\hline Hostility & $0.253^{* *}$ & $-0.230^{* *}$ & $0.227^{* *}$ & \\
\hline Perfectionism & $-0.290^{* *}$ & $0.416^{* *}$ & $-0.123^{* *}$ & $0.138^{* *}$ \\
\hline
\end{tabular}

Chronic fatigue
0.260 (Loneliness) + 0.120 (Hostility)
0.376 (General self-efficacy) + 0.116 (Perfectionism)

Conclusion: Perfectionism might be adaptive effects in well-educated young adults.

They might regard the Perfectionism as self-oriented rather than forced socially prescribed one. Self-oriented Perfectionism might have stress-buffering effect as adaptive emotional intelligence (Lea et al, 2019).

References: Shafran et al, Perfectionism and psychopathology: A review of research and treatment, 2001. Jaremka et al, Pain, depression, and fatigue: loneliness as a longitudinal risk factor 2014. Wang et al, The dual model of perfectionism and depression among Chinese University students, 2017. Lea et al., Does emotional intelligence buffer the effects of acute stress? A systematic review, 2019. 